\title{
science
}
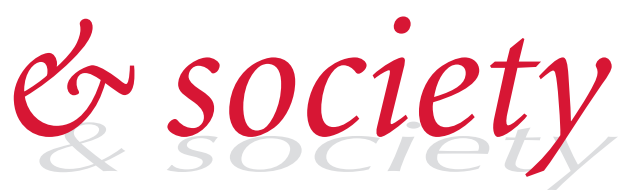

\section{The future of life}

\author{
Creating natural, artificial, synthetic and virtual organisms
}

\section{Ian Pearson}

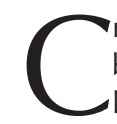

reating life would surely be one of the biggest scientific breakthroughs manthat this breakthrough will be made within the next few years. We are already able to synthesize simple viruses by taking their genetic code from online databases and reconstructing their genomes (Cello et al, 2002). The next few years will almost certainly see the creation 'from scratch' of the first bacterium-again, by using off-the-shelf components and appropriate assembly instructions-and progress will not stop at existing biological techniques. The intent of some researchers is to develop a toolkit of 'synthetic biology' using an engineering approach to reconstruct and redesign vast swathes of biology, and to create a man-made version of 'nature'.

\section{...new life forms will not necessarily be restricted to standard biology [...] they might also be electronic, for example, or conventionally biological, but based on different biochemistry}

The discovery of restriction enzymes and the invention of the polymerase chain reaction enabled specific genes to be cloned and gave biologists the first tools to modify and manipulate living organisms at the molecular level. It is likely that scientists will further refine these tools, and increase their capabilities over the coming decades as they gain more information about biological and biochemical processes from genomics and proteomics. Earth is teeming with life, but the countless number of species that inhabit our planet all rely on the same basic biochemical processes. In my view, this situation will change markedly during the next century, as humans understand increasingly how life works, and learn how to replicate and manipulate it. These new life forms will not necessarily be restricted to standard biology - even if biology acts as an inspiration for their makers - they might also be electronic, for example, or conventionally biological, but based on different biochemistry. Of course, the potential for totally new life forms will expand exponentially once cyberspace and artificial intelligence (Al) are added into the mix of electronics, synthetic and real biology, and as nanotechnology improves and extends the toolkit-both to work out how nature does things and then to improve on it.

A Ithough progress in developing $\mathrm{Al}$ is slower than was expected 40 years ago, it is still ongoing, and it is likely that we will have conscious and intelligent machines some time around 2020. Machine intelligence and consciousness will inevitably be different to our own human intelligence and there will be a lot of debate as to whether conscious machines are 'alive', and what their rights and responsibilities should be. Sadly, there is little evidence that human nature has changed much since Roman times, when one of the forms of public entertainment was watching people hack each other to death in gladiatorial combat. Today, we have Robot Wars, in which remote-controlled machines do battle. The machines are clearly just machines, so there is no debate yet about their treatment. But future robots might have powerful $\mathrm{Al}$, and some will be designed to look and feel just like real people, with polymer muscles covered in silicone rubber. We cannot be sure whether these will ever be used in Robot Wars; however, it would certainly be a great crowd-pleaser if they were human-like-with synthetic blood-the more gore the better as far as audience ratings are concerned. Many will argue that there is no moral problem with using $\mathrm{Al}$ robots in this manner because they are 'just machines'. But if we do allow such use of androids, even if they do not have full consciousness, we will have stooped once again to the lowest level of human morality.

\section{...the potential for totally new life forms will expand exponentially once cyberspace and artificial intelligence are added into the mix of electronics, synthetic and real biology...}

One of the most popular computer games is The Sims ${ }^{\mathrm{TM}}$, produced by EA Games. It allows players to design and orchestrate a virtual soap opera. The player designs the environment, the buildings, the interiors, as well as both the appearance and personality of the characters, and then interferes at will in every aspect of their virtual lives. It is highly compelling and has the ethical advantage of being creative rather than destructive. Of course, the characters only have a tiny amount of $\mathrm{Al}$, but each upgrade brings more. We should expect that similar games in the far future could invoke characters with strong $\mathrm{Al}$ foundations that give them real consciousness and intelligence. Again this raises ethical issues. What level of consciousness or sentience should a game character be allowed before it is given some basic rights and protection against suffering? Will we even be able to measure such things by the time we can create them? Should children be allowed to control conscious beings? Furthermore, smart 'Sims' might start producing saleable goods_-such as software 


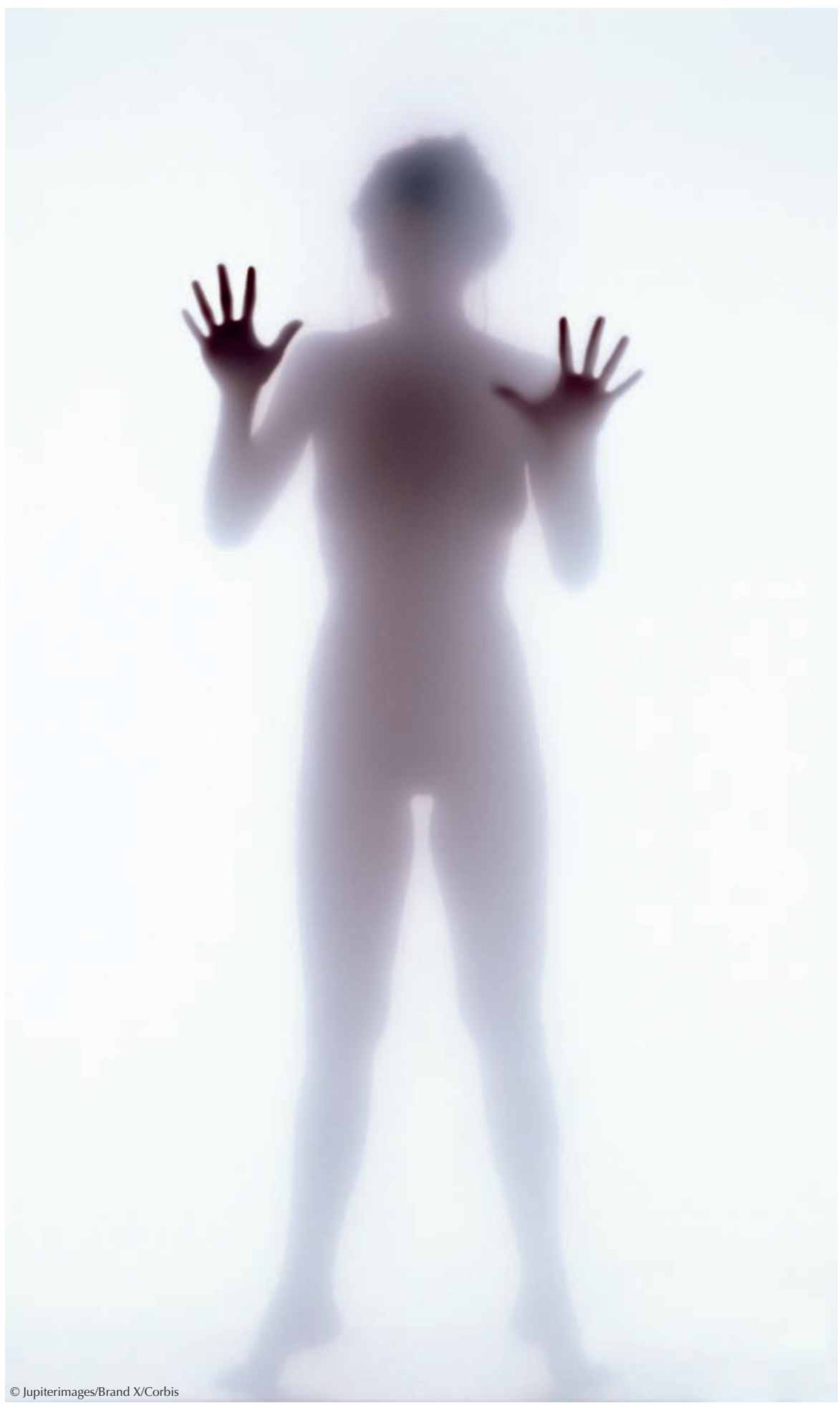

or music - to sell online and make money, which they could invest in a 'timeshare robot' to migrate into the real world. I do not think that governments are quite ready for dealing with immigration from cyberspace. cells to assemble nanostructures such as small molecular clusters or tiny electronic circuits. Meanwhile, the development of molecular switches is accelerating, along with molecular sensing technology - as is, of course, the use of carbon nanotubes to connect components. Such bottom-up assembly is often hailed as the natural replacement for today's lithography. So far, it is presumed that the required assembly would be achieved by tiny machines not by biological cells. However, the assembly of simple circuits by DNA in a test tube has already been demonstrated (Kerren et al, 2003). Perhaps it will become feasible to do this inside living cells by using customized DNA. If and when bacteria can be genetically modified to assemble circuitry, it will be a major breakthrough; once the circuits are assembled, the bacteria could be disposed of, leaving the circuits in place. Another possibility would be that the circuitry could stay inside a bacterium and be powered by its own molecular powerhouse. In a decade or two, there could well be bacteria that enclose fully functioning electronic circuits. Although the circuitry within each cell might be limited, selforganization or biofilms could link many bacteria together into useful computing, storage or sensing devices. These bacteria would self-replicate naturally with their computing power growing organically. It might become possible to grow very large and powerful computers in this way, with the traditional problems of power supply and heat dissipation taken care of directly by nature. By using an evolutionary design methodology, it might be possible to program a large cluster for consciousness.

Furthermore, once we establish bacterial computers that can be networked directly, it would be a relatively trivial step to network them across the entire world through the Internet, thus making global organisms. Further even to this, it then becomes possible to use online intelligence as part of the organism's system, making for truly hybrid organic-electronic-software organisms. This concept of hybridizing organisms - to have a dual existence in both the physical and electronic world-is extremely thoughtprovoking and begs the question of what limits there might be to future life, if any. Such life forms could not only have a wide variety of physical forms, but also an infinite variety of online or cyberspace forms. The cyberspace world is not physically continuous in the same way as the physical universe, either in time or space; there are many disconnected islands, 
and things can pop in and out of existence at different times and locations.

$\mathrm{B}$ iologists can already accomplish limited genetic modification (GM) and genetic selection of embryos. In due course, they will be able to customize many characteristics of our offspring, as well as editing and designing other species. Although it is currently considered unethical to modify human embryos for the purposes of enhancement, in the sufficiently far future, it might be considered irresponsible parenting not to give children the best possible genetic and proteomic start in life.

It might also become feasible to recreate extinct species by using derivatives of cloning and GM technology, provided that highquality samples of DNA are available. Later, we might be able to fill in the gaps where only incomplete samples of DNA are available by using educated guesswork and some 'off-the-shelf' assembly. The first example of a species brought back from extinction by such a technique seems likely before 2010 . There have already been attempts to clone the Tasmanian tiger, thought to have gone extinct in the twentieth century, but it cannot be too long before we are able to do this with any animal whenever good tissue samples are available. Although Jurassic Park might remain in the realms of science fiction, we will eventually have at least the theoretical capacity to rebuild and repopulate the rain forests.

But why stick with historically 'natural' organisms? Eventually, it might become standard practice to blend the characteristics of different species to make organisms that do not exist, and never have existed, in nature. There could be a high demand for creatures such as Furbies to be kept as live pets-rather than electronic toys-and biologists or engineers might design both the appearance and behaviour of new organisms. These could then be introduced into existing natural systems or even into wholly synthetic ecosystems. Even if rain forests have been totally destroyed and their species lost in the future, humans could design and build new ones-perhaps optimized for $\mathrm{CO}_{2}$ fixation, or to be prettier or more interesting - to perpetuate life on earth long after we have gone extinct. We might not go all the way to replacing nature completely, but it seems inevitable that the future organic world will be a combination of natural and synthetic life forms. Nature will certainly become ever more harnessed to human goals.
F or less than $\$ 1,000$, it will be possible to have your full genome sequenced in 2010. A computer program could combine your listing with a friend's listing to produce any number of genetically favourable and unique potential children. Celebrities could combine their genetic listings to produce collectable 'eBaybies' whose genetic sequences could then be auctioned on eBay $\mathbb{B}$ to the highest bidder. Each of these listings would represent a potential future human and, once we have the technology, it will be possible to assemble the required DNA and implant a real embryo into the bidwinning mother. In the further future, it might even be possible to simulate the likely phenotype of the offspring so that parents can choose which 'version' of their child they would like to make a reality. Although 'digital conception' could be feasible any time soon, it will not be possible to use the data to create a real embryo until some time after 2020. These issues are still at least a decade away, but it is time to start serious discussion about them now.

$\mathrm{H}$

umans are embarking on an exciting journey with the power to create new kinds of life. This is not something that should be undertaken lightly. In the future we might be able to recreate extinct species, customize existing organisms, and design and build new organisms, which might coexist with natural ones in the same ecosystems. Similarly, engineers and biologists are already at work on artificial life in cyberspace, with the aim to eventually create conscious software that matches or even exceeds the mental abilities of humans.

Editorial control over nature would make humans the masters of all evolution. Yet if this is ever the case, will such power be used for the good of nature, or the good of the market? It is sad to say, but we will almost certainly gain the required technology many years before we reach the level of cultural sophistication that would ensure the power is wielded with appropriate wisdom; it is going to be like giving a powerful chemistry set to a child for its third birthday.

At the moment, there is far too little discussion of the ethical, moral and technological issues that these possibilities raise, both in academic circles and in the public domain. These abilities, and how to use them, are not something that should be decided by big biotech companies and a few ethicists - the whole population needs to be engaged. Moreover, the debate certainly should not be had in only one country without discussion in the global community. Once synthetic life is here, its impact will be permanent and no country should be allowed to inflict such changes without proper consent. The ethical, legal and practical issues arising from conscious machines, synthetic biology and networked or hybrid organisms are numerous and it will take a long time to evaluate them sensibly. Yet, the technological ability to do these things will be achieved over the next two decades-long before we will be ready to deal with the implications, in my view. Therefore, it is time to start discussion in earnest now.

\section{REFERENCES}

Cello J, Paul AV, Wimmer E (2002) Chemical synthesis of poliovirus cDNA: generation of infectious virus in the absence of natural template. Science 297: 1016-1018

Kerren K, Berman RS, Buchstab E, Sivan U,

Braun E (2003) DNA-templated carbon nanotube field-effect transistor. Science $\mathbf{3 0 2}$ : 1380-1382

This article is a modified version of an article originally published in Nano Magazine in 2007. With kind permission from Nano Magazine, Stirling, UK.

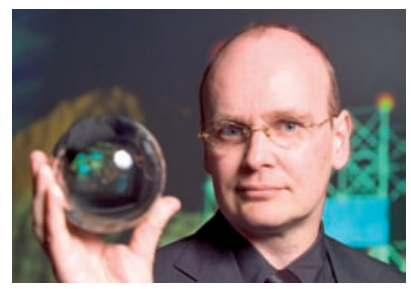

Ian Pearson graduated in 1981 in Applied Mathematics and Theoretical Physics from Queens University in Belfast, Ireland. He joined British Telecommunications Laboratories as a performance analyst, and later worked in network design, computer evolution, cybernetics and mobile systems. From 1991 until 2007, he was BT's Futurologist, tracking and predicting new developments throughout information technology, considering both technological and social implications. He now does exactly the same for Futurizon, a start-up futures institute. As a futurologist and consultant, he lectures and writes on topics such as machine consciousness, human evolution, women's issues, ageing, social trends and advanced computing technology. Pearson is a Chartered Fellow of the British Computer Society, the World Academy of Art and Science, the Royal Society of Arts, the Institute of Nanotechnology and the World Innovation Foundation. He was recently awarded an Honorary Doctor of Science degree by the University of Westminster. E-mail: idpearson@gmail.com

doi:10.1038/embor.2008.62 